Meta

Journal des traducteurs

Translators' Journal

\title{
Linguistique comparative, linguistique impliquée et traduction
}

\section{K.-Richard Bausch}

Volume 16, numéro 1-2, mars 1971

Actes du colloque international de linguistique et de traduction.

Montréal, 30 septembre - 3 octobre 1970

URI : https://id.erudit.org/iderudit/002010ar

DOI : https://doi.org/10.7202/002010ar

Aller au sommaire du numéro

Éditeur(s)

Les Presses de l'Université de Montréal

ISSN

0026-0452 (imprimé)

1492-1421 (numérique)

Découvrir la revue

Citer cet article

Bausch, K.-R. (1971). Linguistique comparative, linguistique impliquée et traduction. Meta, 16(1-2), 45-55. https://doi.org/10.7202/002010ar d'utilisation que vous pouvez consulter en ligne. 


\section{Linguistique comparative, linguistique appliquée et traduction}

La linguistique de nos jours, me semble-t-il, s'apprête, consciemment ou inconsciemment, à se construire une nouvelle Tour de Babel terminologique. Même si l'on se limite à la linguistique comparative (synchronique, bien entendu), citée en premier dans le titre de notre article, il est' aujourd'hui extrêmement facile, en s'appuyant sur les travaux de cette discipline, de démontrer quelle diversité, voire quelle confusion y règnent aussi bien en ce qui concerne les termes employés que les notions et méthodes présentées.

Ainsi, là où les représentants actuels de l'école de Prague, comme E. Benes, V. Fried et V. Schwanzer, emploient le terme de «comparaison analytique » (analytischer Sprachvergleich ${ }^{1}$ ), et que d'autres auteurs parlent de «typologie ${ }^{2}$ » ou tout simplement de "linguistique comparative» (vergleichende Sprachwissenschaft ${ }^{3}$ ), ou encore de «stylistique comparée ${ }^{4} \gg$ ou bien de 《linguistique différentielle ${ }^{5} »$, H. A. Hatzfeld, lui, a forgé l'expression d'idiomatology ${ }^{6}$, d'autres

1. Cf. par exemple E. Beneš, "Das deutsche Passiv im Vergleich mit dem Tschechischen », Probleme der kontrastiven Grammatik. Schriften des Instituts für deutsche Sprache in Mannheim, vol. VIII, Jahrbuch 1969, Düsseldorf, 1970, p. 107 ; V. Fried, "Contrastive Linguistics und analytischer Sprachvergleich ", Linguistische und methodologische Probleme einer spezialsprachlichen Ausbildung (J. Schilling, ed.), Halle, 1967, p. 24-32; V. Schwanzer, "Bemerkungen zur Konfrontation deutscher und slawischer Verbalformen und Verbalstrukturen », Probleme der kontrastiven Grammatik. Schriften des Instituts für deutsche Sprache in Mannheim, vol. VIII, Jahrbuch 1969, Düsseldorf, 1970, p. 126.

2. Cf. à ce propos l'analyse critique de G. Jäger, "Ubersetzungswissenschaft und vergleichende Sprachwissenschaft ", Probleme der strukturellen Grammatik und Semantik (R. Rưžička, ed.), Leipzig, 1968, p. 211.

3. Cf. par exemple M. Wandruszka, "Deutsche und romanische Verbalstrukturen», Probleme der kontrastiven Grammatik. Schriften des Instituts für deutsche Sprache in Mannheim, vol. VIII, Jahrbuch 1969, Düsseldorf, 1970, p. 68.

4. A. Malblanc, Pour une stylistique comparée du français et de l'allemand, Paris, 1944; J.-P. Vinay et J. Darbelnet, Stylistique comparée du français et de l'anglais. Méthode de traduction, Paris, 1966; A. Malblanc, Stylistique comparée du français et de l'allemand. Essai de représentation linguistique comparée et étude de traduction, Paris, 1966.

5. C'est J. Darbelnet qui, pour la première fois, à ma connaissance, a introduit ce terme en français (cf. l'article "Sémantique différentielle", The Canadian Journal of Linguistics/ la Revue canadienne de linguistique, 10 (1964), p. 7-14).

6. Cf. A Critical Bibliography of the New Stylistics Applied to the Romance Literature 1953-1965, Chapel Hill, 1966, p. 157. 
linguistes parlent même de "caractérologie » (Charakterologie ${ }^{7}$ ), H. L. Kufner a écrit un contrastive sketch ${ }^{8}$,W. F. Mackey quelques pages sur les principes d'une differential grammar et d'une differential stylistics ${ }^{9}$; quant à $\mathrm{L}$. Zabrocki, il distingue la «linguistique confrontative typologique» (typologisch vergleichende Sprachwissenschaft) de la "grammaire confrontative» (konfrontative Gramma$\left.t i k^{10}\right)$, il entend par cette dernière ce que la majorité des auteurs américains et européens appellent aujourd'hui applied contrastive linguistics et ce que M. A. K. Halliday appelle tour à tour comparative descriptive linguistics et contrastive linguistics ${ }^{11}$. Il me serait aisé de poursuivre cette énumération, mais, à mon avis, il faut chercher l'explication générale de ce chaos terminologique et conceptuel dans les faits suivants :

1) Ces dernières années, on constate une véritable explosion de recherches qui se disent toutes, d'une manière ou d'une autre, «contrastives ${ }^{12}$ » et qui traitent synchroniquement de problèmes interlinguaux. Pour étayer cette affirmation, je me permets de citer, sans pouvoir entrer dans les détails, bien sûr, les trois phénomènes que voici :

a) Divers projets nationaux de «grammaires contrastives » ont vu le jour. Ainsi au Center for Applied Linguistics de Washington, on élabore depuis 1959 la Contrastive Structure Series, opposant l'anglo-américain respectivement à l'allemand, à l'espagnol, à l'italien, au français et au russe ; on envisage également depuis peu la rédaction d'autres grammaires, opposant l'anglo-américain aux langues scandinaves et africaines ${ }^{13}$. - Des projets semblables ont été entrepris pour l'allemand et l'anglais en 1968 à Stuttgart ${ }^{14}$, à Zagreb, également depuis 1968, pour le serbo-croate et l'anglais ${ }^{15}$, à Poznán depuis 1969 pour le polonais

7. Cf. par exemple l'article de G. Jäger, «Übersetzungswissenschaft und vergleichende Sprachwissenschaft 》, Probleme der struckturellen Grammatik und Semantik ( $\mathrm{R}$. Rủžička, ed.), Leipzig, 1968, p. 212.

8. Cf. The Grammatical Structures of English and German. A Contrastive Sketch, Chicago, 1962.

9. Cf. Language Teaching Analysis, Londres, 1965, p. 88-92 et p. 95-96; cf. en plus, les autres chapitres sur * Differential Phonetics» et "Differential Lexicology ».

10. Cf. "Grundfragen der konfrontativen Grammatik ", Probleme der kontrastiven Grammatik. Schriften des Instituts für deutsche Sprache in Mannheim, vol. VIII, Jahrbuch 1969, Düsseldorf, 1970, p. 31-52.

11. Cf. "Comparison and Translation", The Linguistic Sciences and Language Teaching (M. A. K. Halliday, A. McIntosh, P. Strevens, ed.), Londres, 1964, p. 111-134.

12. On est donc aujourd'hui un peu à l'opposé de ce que E. Kleinjans avait écrit il y a douze ans : "The work of comparing two languages for the purpose of finding those points in the language being learned that will cause interference for a speaker of the other language is of recent origin. There is therefore but a small amount of literature on the subject " (cf. A Descriptive-comparative Study Predicting Interference for Japanese in Learning English Noun-headed Modification Patterns, traduit et annoté par Tadashi Ito, Tokyo, 1958, p. 171).

13. Cf. le plan d'ensemble avec toutes les références bibliographiques nécessaires de W. Nemser : "Contrastive Linguistics at the Center for Applied Linguistics》 (à paraître dans The Linguistic Reporter).

14. Cf. maintenant le tour d'horizon du « Projekt für angwandte kontrastive Sprachwissenschaft (PAKS) " de G. Nickel : "The Applied Contrastive Linguistics Project of the University of Stuttgart \#, The Linguistic Reporter, 12 (1970), p. 4-7.

15. Cf. la vue d'ensemble de R. Filipovič : "The Yugoslav Serbo-Croatian - English Contrastive Project» (à paraitre dans les actes du 2 nd International Congress of Applied Linguistics, Cambridge, 1969) ; cf. aussi les Abstracts du congrès, Cambridge, 1969, p. 52. 
et l'anglais ${ }^{16}$, et depuis quelques mois à Bucarest pour le roumain et l'anglais ${ }^{17}$; $b$ ) De plus, ont paru des bibliographies spéciales consacrées aux travaux en linguistique contrastive, comme celle de J. H. Hammer et F. A. Rice ${ }^{18}$, qui a été mise à jour par $\mathbf{R}$. Thiem ${ }^{19}$;

c) Et enfin différents congrès internationaux ont été organisés, qui visent avant tout à la discussion des méthodes contrastives. Ainsi, par exemple, le $19^{\text {th }}$ Round Table Meeting on Contrastive Linguistics and its Pedagogical Implications à Washington en $1968^{20}$, la session de printemps de l'Institut für deutsche Sprache à Mannheim en $1969^{21}$, la section "Contrastive Linguistics», la plus importante du $2^{e}$ Congrès international de linguistique appliquée à Cambridge en $1969^{22}$ et enfin la Pacific Conference on Contrastive Linguistics and Language Universals qui vient d'avoir lieu à Hawaii.

2) Un autrc élément contribuant à expliquer le désordre terminologique, est que l'on peut, çà et là, constater une tendance à ignorer les relations historiques entre la notion «contrastive » et les concepts et méthodes déjà développés par d'autres linguistes ou comparatistes ou contrastistes ${ }^{23}$. En guise d'illustration, je voudrais brièvement citer les deux exemples suivants :

a) Dans tous les ouvrages et articles publiés par le Center for Applied Linguistics de Washington, on cherche en vain la discussion par exemple de la conception théorique de ce qu'on est convenu d'appeler «comparaison analytique », conception que les membres de l'école de Prague, en s'opposant d'ailleurs expressis verbis à la méthode historico-génétique des néo-grammairiens, ont élaborée systématiquement il y a quarante ans. Dans ce contexte, je me contente de citer B. Trnka qui, dans son rapport, communiqué au $1^{\text {er }}$ Congrès des philologues slaves et publié en $1929^{24}$, écrivait :

16. Cf. L. Zabrocki, "Grundfragen der konfrontativen Grammatik ", Probleme der kontrastiven Grammatik. Schriften des Instituts für deutsche Sprache in Mannheim, vol. VIII, Jahrbuch 1969, Düsseldorf, 1970, p. 31-52.

17. Cf. W. Nemser, "Contrastive Linguistics at the Center for Applied Linguistics 》, et en plus, W. Nemser et $T$. Slama-Cazacu, "A Contribution to Contrastive Linguistics (A Psycholinguistic Approach : Contact Analysis)", Revue roumaine de linguistique, vol. XV (1970), p. 101-128.

18. Cf. A Bibliography of Contrastive Linguistics, Washington (D.C.), 1965.

19. Cf. "Bibliography of Contrastive Linguistics », PAKS-Arbeitsbericht (Stuttgart), 2 (1969), p. 79-96, et 3-4 (1969), p. 93-120.

20. Cf. les actes qui ont été publiés dans Monograph Series on Languages and Linguistics, vol. XXI (J. E. Alatis, ed.), Washington (D.C.), 1968.

21. Cf. les actes Probleme der kontrastiven Grammatik. Schriften des Instituts für deutsche Sprache in Mannheim, Düsseldorf, 1970.

22. Cf. les actes qui paraîtront en 1971 .

23. Bien sûr, il y a des exceptions, par exemple R. Filipovič, "Contrastative Analysis of Serbo-Croatian and English ", Studia Romanica et Anglica Zagrabiensia, 23 (1967) p. 5-27 ou par exemple G. Nickel, «Bericht über Ergebnisse der kontrastiven Analyse sprachlicher Phänomene im Deutschen und Englischen», Deutschunterricht fü̈ Ausländer, 1968, p. 140-152.

24. Cf. « Méthode de comparaison analytique et grammaire comparée historique », Travaux du Cercle linguistique de Prague, vol. I : Mélanges dédiés au premier Congrès de philologues slaves, Prague, 1929, p. 33-38; cf. en plus V. Mathesius, " On Some Problems of the Systematic Analysis of Grammar ", Travaux du Cercle linguistique de Prague, vol. VI : Etudes dédiées au IVe Congrès de linguistes, Prague, 1936, p. 95-107. C'est d'ailleurs V. Mathesius qui, pour la première fois, a essayé d'exploiter cette méthode en vue d'une application à l'enseignement soit de la langue maternelle soit d'une langue étrangère; cf. à ce propos l'analyse de V. Fried, "Comparative Linguistic Analysis in Language Teaching ", Modern Language Teaching. Papers from the 9th Congress of the Fédération internationale des professeurs de langues vivantes (H. Jalling, ed.), Londres, 1968, p. 38-46. 
La méthode de comparaison analytique ou synchronique admet [...] la possibilité de la comparaison d'états de langue entre langues apparentées de loin seulement ou pas du tout apparentées, et permet ainsi de constater des tendances linguistiques et des catégories grammaticales plus générales, constatations que l'on n'aurait pu faire par la grammaire historique des langues d'un même groupe.

À l'origine, cette méthode analytique ne poursuit donc qu'un but comparatif au sens fort du terme, relevant entièrement de la linguistique «pure ». Ce qui étonne, c'est que, par exemple dans l'introduction générale aux différentes monographies, l'éditeur général de la Contrastive Structure Series, C. A. Ferguson, vise exactement le même but sous le terme de contrastive analysis, sans se référer aux travaux de l'école de Prague (pourtant bien connus) et malgré le caractère nettement pratique de toute la série ${ }^{25}$;

b) Le terme technique de contrastive linguistics apparaît pour la première fois, non pas dans la Contrastive Structure Series, mais dans un petit article de G. L. Trager, paru à Oklahoma en 1949 sous le titre « The Field of Linguistics ${ }^{26}$ ». En quelques pages seulement, G. L. Trager expose la conception d'une linguistique contrastive générale qui, d'une part, en opposition à la linguistique descriptive, met, comme le fait l'école de Prague, la comparaison synchronique de différentes langues apparentées ou non apparentées au centre de sa théorie, mais qui exclut d'autre part toute idée d'application pratique. Ni les membres du centre américain, ni les représentants des différentes écoles contrastives européennes ne semblent avoir connu cette conception. C'est à mon avis la seule hypothèse qui puisse expliquer que - malgré les différents postulats purement linguistiques - le terme et la notion de «linguistique contrastive » aient pu dès le début s'orienter très nettement vers des objectifs d'application. L'influence des idées que C. C. Fries avait déjà développées dans cette direction en 1945 dans son manuel Teaching and Learning English as a Foreign Language ${ }^{27}$, est aujourd'hui tout à fait reconnue. De même on voit en général dans l'ouvrage de R. Lado, Linguistics across Cultures. Applied Linguistics for Language Teachers ${ }^{28}$, l'amorce de l'évolution systématique de la linguistique contrastive dite « appliquée ».

II

Dans ce qui suit, je voudrais tout d'abord essayer de caractériser et de délimiter les interdépendances et les différences les plus marquantes que l'on peut établir entre les notions et méthodes de la linguistique comparative analytique ou synchronique d'une part, et la linguistique contrastive (appliquée) d'autre part ${ }^{29}$; ceci me semble être d'une nécessité absolue afin de pouvoir esquisser

25. Bien entendu, je parle dans ce contexte de "but» et non pas de « méthode ". On peut se référer pour les différences méthodiques entre les deux écoles à V. Fried, «Der analytische Sprachvergleich und die Methodik des Fremdsprachenunterrichts", Fremdsprachenunterricht in unserer Zeit, Sonderheft zu Praxis des neusprachlichen Unterrichts, 1965 , p. 67-74.

26. Studies in Linguistics : Occasional Papers, no 1, 2e éd., Oklahoma, 1950. Je dois cette référence au cours de E. Coseriu fait en 1970 à l'Université de Tübingen.

27. Ann Arbor, particulièrement p. 9.

28. Ann Arbor, 1957.

29. Cf. également W. Nemser et T. Slama-Cazacu, "A Contribution to Contrastive Linguistics (A Psycholinguistic Approach : Contact Analysis) ", Revue roumaine de linguistique, vol, XV (1970), p. 108 : «... the crucial differences between contrastive linguistics and comparative typology have not been generally recognized.» 
ensuite les rapports respectifs des deux linguistiques avec la traduction. Tout ce que je vais dire fait d'ailleurs partie d'une recherche épistémologique détaillée qui est en cours et dont je ne peux, hic et nunc, donner bien sûr qu'un bref aperçu $^{30}$.

1) La linguistique contrastive appliquée est déterminée, je viens de l'indiquer, par des phénomènes extralinguistiques; je m'explique : elle essaie en fin de compte de faciliter et de rationaliser systématiquement l'enseignement d'une langue étrangère; par conséquent, la linguistique contrastive dépasse, par son orientation même, les limites de la méthode comparative analytique et doit être classée dans le cadre de la linguistique appliquée; en d'autres termes, si l'on admet que chaque science peut être divisée en une branche théorique ou pure et une branche pratique ou appliquée, il en résulte que la linguistique contrastive n'est rien d'autre que l'exploitation des résultats de la linguistique comparative synchronique en vue d'une application ${ }^{\mathbf{3}}$.

Je voudrais proposer de distinguer rigoureusement les deux disciplines à l'avenir sur les plans notionnel et terminologique, si l'on veut dans le sens où l'entend L. Zabrocki : partant d'un autre point de vue, il aboutit à la classification en une linguistique confrontative d'une part, conçue sur le plan de la typologie, et d'autre part une grammaire confrontative appliquée à l'enseignement concret de l'anglais aux Polonais ${ }^{32}$.

2) La linguistique contrastive appliquée s'occupe des interférences interstructurales, c'est-à-dire des transferts négatifs de certaines habitudes linguistiques de la langue maternelle à la langue étrangère ${ }^{33}$. Les « fautes », qui en résultent, ne sont donc que linguistiquement motivées, quoiqu'il soit bien évident qu'il existe en pratique chez l'élève d'autres sources d'erreurs, par exemple de nature psychologique, etc., qui dépassent, bien sûr, le cadre de toute recherche contrastive. À mon avis, il faut donc, contrairement aux opinions d'un certain nombre de contrastistes, reconnaître que les deux termes de «fautes » d'une part et d' 'interférences » d'autre part ne sont que partiellement synonymes.

Les interférences négatives sont causées par les divergences partielles ou totales qui existent entre les structures des différentes langues comparées ${ }^{34}$. Donc, l'analyse contrastive est (ou même «doit » être), en tant qu'analyse linguistique, déjà marquée par les éléments entrant en ligne de compte comme causes possibles

30. J'espère pouvoir terminer ce travail en 1971.

31. Cf. également L. Spalatin, "Contrastive Methods", Studia Romanica et Anglica Zagrabiensia, 23 (1967), p. 29: "Contrastive linguistics is conceived here as primarily concerned with the practical purpose of making a second language more easily accessible to speakers of another language. Conceived in this way, contrastive linguistics is applied comparative descriptive linguistics.

32. Cf. "Grundfragen der konfrontativen Grammatik», Probleme der kontrastiven Grammatik. Schriften des Instituts für deutsche Sprache in Mannheim, vol. VIII, Jahrbuch 1969, Düisseldorf, 1970, p. 31-52.

33. En syntaxe traditionnelle on a habituellement qualifié le même phénomène de "germanismes ", « anglicismes ", etc.

34. Bien sûr, il y a également des interférences "positives » causées par les convergences totales interstructurales qui ne posent pas le moindre learning problem et qui, au contraire, favorisent le processus d'apprentissage. A ce propos $\mathrm{cf}$. la classification en différents types de divergences dans W. Nemser et V. Ivir, "Research Guide for Project Workers : Morphology and Syntax ", The Yugoslav Serbo-Croatian - English Contrastive Project. A. Reports 1, Zagreb, 1969 , p. 4 . 
d'interférences; cela signifie qu'elle néglige automatiquement — et ceci à juste titre - la confrontation des convergences interlinguales et qu'elle est donc descriptivement plus ou moins incomplète ${ }^{35}$.

Il est clair que la linguistique comparative synchronique, prise au sens strict du terme, devrait absolument rejeter une telle méthode. En effet, elle doit au contraire s'efforcer d'arriver à des conclusions qui prennent également en considération les convergences, afin de pouvoir rendre compte descriptivement dans les moindres détails des réalités linguistiques des langues comparées.

Puisqu'un grand nombre de linguistes appellent leurs études à ce propos expressis verbis, je le répète, contrastives et appliquées, tout en ayant l'ambition d'entreprendre dans les mêmes analyses, des recherches «typologiques ${ }^{36}{ }$ il paraît évident qu'en fin de compte ce sont les résultats linguistiques qui vont en souffrir.

3) L'interférence linguistique se produit selon le principe dit «unilatéral » ou « unidirectionnel ${ }^{37} \gg$; en d'autres termes, l'interférence concrète est liée au processus d'apprentissage qui va dynamiquement de la langue maternelle à la langue étrangère; elle est en principe irréversible. Ainsi par exemple pour un Anglais qui apprend à se servir de l'article défini en allemand, le risque de confondre le masculin avec le féminin, le neutre avec le masculin, etc., est extrêmement grand, puisqu'il y a divergence partielle entre les deux structures; inversement, pour un Allemand qui apprend à utiliser l'article anglais, le risque d'interférences « linguistiques » à partir de l'allemand n'existe pas.

Il en résulte qu'une grammaire contrastive doit être, elle aussi, «unilatérale » et rendre compte du caractère dynamique du processus ${ }^{38}$, tandis qu'une grammaire «bilatérale 》 ou «réciproque 》 - a two-way contrastive grammar -

35. Comme exemples je cite d'abord la thèse de R. P. Stockwell, $A$ Contrastive Analysis of English and Tagalog, Los Angeles, s. d., qui d'ailleurs a influencé profondément le développement de la linguistique contrastive, cf. p. A-2 : "The analysis itself is a first approximation of what linguists have long maintained should be done in language teaching: a point by point study of the structural differences between two languages..." . Ensuite je m'en réfère au rapport de $\mathbf{R}$. $\mathbf{J}$. di Pietro, « Alcune riflessioni sulla linguistica applicata all'insegnamento » fait au Convegno Internazionale di Studi sul Tema « L'insegnamento dell' Italiano in Italia e fuori di Italia », Rome, 1970, p. 3 du texte polycopié : "Sarebbe inutile insegnare allo studente di lingua inglese quelle forme grammaticali della nuova lingua che a lui sono gia note perchè presenti nella sua. [...] Di qui, l'importanza dell' analisi contrastiva della lingua insegnata e di quella degli studenti corne mezzo per arrivare alla scoperta delle differenze tra le competenze ideali delle due lingue. $\gg$

36. A ce propos, je me contente de citer un seul exemple à savoir l'opinion de J. Czochralsky, " Grundsätzliches zur Theorie der kontrastiven Grammatik ", Linguistics, 24 (1966), p. 25 : "Ein kontrastiver Vergleich, [...] kann eine tiefere Einsicht in das Funktionieren des Systems gewähren. »

37. Cf. J. Czochralsky, «Grundsätzliches zur Theorie der kontrastive Grammatik», Linguistics, 24 (1966), p. 18 ; cf. en plus L. Spalatin, "Contrastive Methods", Studia Romanica et Anglica Zagrabiensia, 23 (1967), p. 28, ou bien R. P. Stockwell et J. D. Bowen, The Sounds of English and Spanish, Chicago, 1965, p. 7 : "... the trip from one language into another is taken on a one-way street"; cf. aussi $\dot{\mathrm{E}}$. A. Levenston, "The Translation — Paradigma Technique for Contrastive Syntax », IRAL, vol. III (1965), p. 221-225.

38. Comme exemple typique je ne cite que l'ouvrage de M. Hashimoto, From Japanese to English. A Contrastive Analysis Based on a Transformational Model, Ph. D. Dissertation in Linguistics, Washington, 1966. 
envisagée par exemple par le centre de Zagreb ${ }^{30}$, qui pourrait être utilisée dans les deux directions avec exactement le même profit, ne pourra au fond, à mon avis, rien donner d'autre que la jonction un peu artificielle de deux grammaires différentes.

Par contre, il ressort clairement de ce qui précède qu'en méthode comparative analytique, «il faut accorder, pour citer $M$. Wandruszka, à tour de rôle [...] la position privilégiée de l'original » à chacune des langues respectives ${ }^{40}$. En transposant ceci dans notre terminologie : l'analyse comparative doit négliger le principe dynamique de 1 '« unidirectionnalité » et devenir « réciproque » et «statique »(two-directional, tri-directional, etc.).

4) Puisque c'est sur l'usage normatif de la langue étrangère que s'aligne le processus d'apprentissage, l'analyse contrastive envisage par conséquent l'élimination à priori des interférences uniquement au niveau de la performance ${ }^{41}$, et ceci, bien sûr, dans tous les plans de la langue, dans la phonologie aussi bien que dans la syntaxe, etc. Là encore, il y a une différence avec les analyses comparatives synchroniques qui, elles, peuvent s'effectuer au niveau de la parole (et de la norme) aussi bien qu'au niveau du système, tout en englobant les différents plans ${ }^{42}$.

Dans ce contexte, il faut dire que toute confrontation de langues présuppose évidemment une description indépendante de chaque langue, selon le même modèle grammatical, bien entendu. Je ne peux que mentionner quelques problèmes qui en résultent : a) À mon avis, la confrontation peut se fonder sur tout modèle de description, à la seule condition qu'il soit suffisamment «explicite $» ; b$ ) Il faut se rendre compte que certains modèles, comme le modèle transformationnel, présentent un net avantage dans l'analyse de la syntaxe tandis qu'à d'autres niveaux, comme la phonologie, on pourrait quand même - et je suis conscient du caractère délicat de ce que je vais affirmer - poser la question de savoir si un modèle taxinomique ne serait pas préférable.

5) L'enseignement d'une langue étrangère, et par conséquent aussi l'analyse contrastive adéquate, est limité à deux langues, la langue maternelle et la langue étrangère ${ }^{43}$; cela signifie : a) Que les résultats concrets obtenus dans une telle language-pair-bound confrontation ne se transposent pas obligatoirement à d'autres langues; des divergences qui jouent par exemple entre l'allemand et l'italien peuvent apparaître comme convergences dans une analyse entre l'allemand et

39. Cf. R. Filipovič, \& Contrastive Analysis of Serbo-Croatian and English », Studia Romanica et Anglica Zagrabiensia, 23 (1967), p. 24 ; et maintenant surtout R. Bugarski, " Direction and Continuity in Contrastive Analysis ", The Yugoslav Serbo-Croatian - English Contrastive Project. A. Reports 1, Zagreb, 1969, p. 9.

40. Cf. Esquisse d'une critique comparée de quelques langues européennes ", Travaux de linguistique et de littérature, vol. V (1967), p. 169.

41. Cf. par exemple à ce propos la classification en mistakes (au niveau de la performance) et errors (au niveau de la compétence) de S. P. Corder, « The Significance of Learner's Errors ", IRAL, vol. V (1967), p. 161-170.

42. Cf. à ce propos aussi E. Coseriu, «Über Leistung und Grenzen der kontrastiven Grammatik ", Probleme der kontrastiven Grammatik. Schriften des Instituts für deutsche Sprache in Mannheim, vol. VIII, Jahrbuch 1969, Düsseldorf, 1970, p. 9-30.

43. Cf. par exemple L. Spalatin, "Contrastive Methods", Studia Romanica et Anglica Zagrabiensia, 23 (1967), p. 29 : "Contrastive linguistics is concerned with only two languages at a time. 》 
l'anglais et vice versa. Bien sûr, cette relativité dépend de la parenté typologique entre les langues en considération; $b$ ) Que la rédaction d'une "grammaire française à l'usage général des étrangers $\gg$ se trouverait en pleine contradiction avec ce principe.

Pour l'analyse comparative analytique par contre, le nombre des langues à comparer est, pour reprendre ce que F. Strohmeier écrivait il y a soixante ans, illimité du moins théoriquement ${ }^{44}$.

Je m'arrête à cette première caractérisation. Cependant, j'en suis conscient, il serait encore possible d'énumérer d'autres traits distinctifs entre la linguistique contrastive appliquée et la méthode de comparaison analytique. Pourtant j'espère, en toute modestie, avoir atteint certains objectifs : tout d'abord avoir montré que tous ceux qui ne voient entre les deux disciplines ainsi délimitées qu'une simple et pure synonymie terminologique ont tort ${ }^{45}$; et ensuite $\mathrm{j}$ 'espère avoir jeté les fondements nécessaires à une description et à un examen des relations de ces deux disciplines avec la traduction.

\section{III}

Le problème de la traduction peut être considéré sous des angles différents dont je me contenterai d'énumérer les plus importants :

1) D'abord, il faut dire un mot sur la position de la stylistique comparée, bien entendu dans la délimitation que je viens d'esquisser :

a) A. Malblanc, J.-P. Vinay et J. Darbelnet envisagent, c'est bien connu, dans leurs stylistiques comparées «bilatérales» le but d'application à l'enseignement de la traduction, et pourtant A. Malblanc par exemple donne en même temps à son ouvrage le sous-titre Essai de représentation linguistique comparée ; donc, parallèlement au but d'application, il poursuit un but nettement linguistique et comparatif. En examinant d'une manière détaillée les exemples que $\mathrm{A}$. Malblanc présente ainsi dans les différents chapitres, on constate qu'il analyse de préférence les divergences et néglige la description des convergences ${ }^{46}$, c'est-à-dire qu'il reste entièrement fidèle au principe que $J$. Darbelnet avait développé à propos d'une «sémantique différentielle ${ }^{47}$ ». En plus, dans tout son ouvrage A. Malblanc compare de préférence les deux langues dans la direction allemand-français, ce qui signifie qu'il respecte, et ceci à juste titre, le caractère « unidirectionnel » du processus de la traduction (cf. ci-après). C'est ainsi que cette stylistique tout entière devrait être qualifiée, en accord avec la partie II de mon article, plutôt de « contrastive» que de « comparative». Or, ce qui a été affirmé précédemment, à savoir qu'il est absolument nécessaire de distinguer entre la linguistique contras-

44. Cf. Der Stil der französischen Sprache, Berlin, 1910, préface.

45. Cf. par exemple J. Lotz, "Introductory Remarks", Contrastive Linguistics and Its Pedagogical Implications, p. 10.

46. J'ai essayé de prouver cette thèse en examinant un certain nombre d'exemples concrets dans mon petit article : "Qualité en traduction et linguistique dite différentielle ", Babel, vol. XVI (1970), p. 13-20.

47. Cf. "Sémantique différentielle", The Canadian Journal of Linguistics/la Revue canadienne de linguistique, 10 (1964), p. 7 : "Peut-être le terme différentielle convient-il mieux pour ce genre d'étude, car il s'agit surtout de dégager des différences. De plus, il a l'avantage d'être assez proche par le sens de contrastive qui est déjà établi dans les ouvrages de linguistique rédigés en anglais. : 
tive appliquée d'une part et la linguistique comparative d'autre part, je tiens à le répéter dans ce contexte : puisqu'une stylistique contrastive, par sa nature même, implique, elle aussi, le danger de falsifier les conclusions d'une véritable comparaison stylistique synchronique entre plusieurs langues ${ }^{48}$, je me permets de proposer d'introduire à l'avenir la même distinction, dans ce domaine aussi ;

b) De ce point de vue, la position de la «critique comparée multilatérale », développée par $M$. Wandruszka ${ }^{49}$, est plus claire : d'abord $M$. Wandruszka renonce à tout but d'application (le mot «interférence » par exemple n'apparaît nulle part dans ses travaux), ensuite il respecte les convergences interstructurales et finalement, pour lui, la traduction n'est pas significative en tant que processus, mais plutôt en tant que "produit » de ce processus ${ }^{50}$. Par conséquent, ses études multilatérales restent entièrement sur le plan comparatif.

2) Du point de vue inverse on peut se demander, et bien sûr on se l'est déjà demandé, si la traduction en tant que produit, qui dépend - comme on sait d'un processus caractérisé essentiellement par des aspects subjectifs, peut vraiment fournir, toujours associée à l'original, une base objective et linguistiquement adéquate pour les analyses respectives, pour ce que R. Filipovic appelle la descriptive translational method ${ }^{51}$. Plusieurs auteurs, par exemple J. Ellis ${ }^{52}$, V. Ivir ${ }^{53}$, T. Krzeszowski ${ }^{54}$, M. Marton ${ }^{55}$, L. Spalatin ${ }^{56}$, M. Wandruszka ${ }^{57}$ et d'autres encore, y compris ma modeste personne, se sont prononcés positivement sur le fond de ce problème.

Pourtant le point donnant lieu à discussion porte sur les modalités selon lesquelles il faut choisir le corpus de traductions afin de chercher à éliminer les aspects subjectifs, que je viens de mentionner. Les conditions essentielles sont à mon avis les suivantes : $a$ ) Il faut se baser sur des textes avec leurs traductions, tout en assimilant les résultats de la linguistique de la parole, et non pas se baser sur des syntagmes, des unités lexicales, etc., isolés du texte intégral ; $b$ ) Les traductions doivent être déjà faites, comme $J$. Ellis le dit, «by practical translators, for some non-metalinguistic purpose $58 \gg ; c$ ) Chaque langue d'arrivée doit devenir

48. Cf. l'exemple que j'ai analysé dans : «Qualité en traduction et linguistique dite différentielle ", Babel, vol. XVI (1970), p. 13-20.

49. Cf. maintenant son opus magnum : Sprachen vergleichbar und unvergleichlich, München, 1969.

50. Cf. la caractérisation détaillée dans K.-R. Bausch, "Ubersetzungswissenschaft und angewandte Sprachwissenschaft. Versuch einer Standortbestimmung » (à paraître dans Lebende Sprachen) ; cf. aussi O. Kade, Zufall und Gesetzmässigkeit in der $\ddot{U} b e r s e t z u n g$, Beihefte zur Zeitschrift Fremdsprachen $n^{\circ}$ I, Leipzig, 1968, particulièrement p. 30 ; et C. James, « Deeper Constrastive Study », IRAL, vol. VII, particulièrement p. $87 \mathrm{ss}$.

51. Cf. "Contrastive Analysis of Serbo-Croatian and English ", Studia Romanica et Anglico Zagrabiensia, 23 (1967), p. 21.

52. Cf. Towards a General Comparative Linguistics, The Hague, 1966.

53. Cf. "Contrasting via Translation : Formal Correspondence vs Translation Equivalence ", The Yugoslav Serbo-Croatian - English Contrastive Project. B. Studies, Zagreb, 1969, p. 13-25.

54. Cf. "Fundamental Principles of Structural Contrastive Studies », Glottodidactica, vol. II (1967), p. 33-39.

55. Cf. "Equivalence and Congruence in Transformational Contrastive Studies», Studia Anglica Posnaniensia, vol. I (1968), p. 53-62.

56. "Contrastive Methods », Studia Romanica et Anglica Zagrabiensia, 23 (1967), p. 29.

57. Sprachen vergleichbar und unvergleichlich, München, 1969.

58. Towards a General Comparative Linguistics, The Hague, 1966. 
langue de départ, ce qui est grosso modo identique à ce que L. Spalatin appelle la backtranslation method ${ }^{59}$; $d$ ) Les traductions doivent être quantitativement représentatives, c'est-à-dire correspondre qualitativement à ce que j'appelle «la norme linguistique comparative ${ }^{60} »$, qui, une fois établie, permet d'éliminer non seulement toutes les traductions fausses univoques, mais aussi - et ceci me semble être plus important - lesdites sous-traductions et surtraductions. Bien entendu, pour pouvoir juger les traductions d'une manière adéquate, on doit relier cette critique linguistique à la détermination la plus exacte possible du type, ou plutôt des différents types de textes auxquels on a affaire ${ }^{61}$.

3) Depuis quelque temps, des linguistes contrastifs commencent à élargir la sphère d'application de leurs analyses et essaient de voir dans la linguistique contrastive une espèce de «Sésame, ouvre-toi » pour un certain nombre d'autres disciplines, particulièrement pour la flesh-and-blood-translation. À ce propos je ne citerai que l'opinion de E. König :

... contrastive investigations should not only be seen in connexion with language teaching but also in connexion with the «science of translation》, which is beginning to develop into an independent discipline within applied linguistics. To a certain degree the aims and interests of contrastive linguistics are identical with those of this new science 62 .

Or, on peut se demander si vraiment une analyse contrastive faite dans l'optique d'une application à l'enseignement d'une langue étrangère, peut à la fois servir de base solide et suffire pour une analyse scientifique du processus de la traduction, bien entendu dans ses aspects linguistiques. En réponse, je voudrais faire les remarques suivantes :

a) Si l'on compare les deux domaines de recherche, c'est-à-dire le processus de traduction et le processus d'apprentissage d'une langue étrangère, on doit d'abord reconnaître qu'il y a un certain nombre de caractéristiques communes, parmi lesquelles les plus importantes sont : le principe dynamique de l'" unidirectionnalité ${ }^{63} »$, le fait que la traduction prend comme point de repère la norme dans la langue d'arrivée, et la limitation du nombre des langues à deux. Malgré ces concordances de détail, la différence est aussi fondamentale qu'évidente : traduire «as an end in itself and not as a means of language learning» - et là j'ai presque honte de prononcer cette vérité de La Palice - n'est possible, bien sûr, qu'au moment où l'apprentissage de la langue étrangère touche, pour ainsi dire, à la native speaker competence; lorsque E. König affirme que les buts et les intérêts de la linguistique contrastive sont plus ou moins identiques avec ceux de la science de la traduction, il porte un jugement pour le moins gratuit;

b) Chaque discipline scientifique - je l'ai dit déjà dans le premier tiers de mon exposé - peut être divisée en une partie théorique ou pure et une partie pratique ou appliquée. Cela s'applique également à la science de la traduction. Ainsi faut-il

59. "Contrastive Methods", Studia Romanica et Anglica Zagrabiensia, 23 (1967), p. 33.

60. "Qualité en traduction et linguistique dite différentielle ", Babel, vol. XVI (1970), p. 16ss.

61. Cf. les derniers essais pour établir une typologie de textes de K. Reiss, "Textbestimmung und Übersetzungsmethode. Entwurf einer Texttypologie», Ruperto-Carola. Zeitschrift der Vereinigung der Freunde der Studentenschaft der Universität Heidelberg e. V., vol. XXI (1969), p. 69-75.

62. Cf. PAKS-Arbeitsbericht, 1 (1968), p. 60-61.

63. Cf. le terme synonyme decision process de J. Levy, "Translation as a Decision Process", To Honor Roman Jakobson, vol. II, The Hague, 1967, p. 1171-1182. 
encore une fois corriger l'opinion de E. König : en effet, la science de la traduction appliquée, par exemple appliquée à l'enseignement efficace de la traduction, a sa place légitime dans la linguistique appliquée. D'autre part l'intégration d'une théorie de la traduction, dans cette discipline ${ }^{64}$, se révèle comme une nette contradictio in adjecto ${ }^{65}$;

c) Répétons finalement qu'une analyse contrastive ainsi qu'une stylistique contrastive, comme le confirme un examen des travaux dans ces domaines, sont essentiellement caractérisées par le poids donné à la description des divergences interstructurales. Soulignons que de telles analyses n'offrent qu'une partie de la base nécessaire et ne respectent pas suffisamment le côté stylistique qui est tout de même présent dans chaque texte traduit, indépendamment du type de texte auquel il se rattache. Je m'explique : là où il y a divergence interstructurale, une analyse contrastive peut parfaitement bêcher et cultiver ce terrain d'abord linguistiquement, c'est-à-dire décrire les servitudes, les automatismes, les procédés de traduction obligatoires — dans ce sens, je serai pleinement d'accord avec J. Darbelnet - et ensuite exploiter ces descriptions en vue de rationaliser et de faciliter l'enseignement de la traduction. Pourtant l'option stylistique, piment de chaque traduction, s'explique dans la grande majorité des exemples concrets et cela tombe sous le sens - exclusivement à la lumière des convergences interlinguales, qui, comme les divergences, font l'objet d'analyses comparatives.

Donc, pour finir, il me reste seulement à appuyer les conceptions théoriques ou pratiques qui considèrent la science de la traduction as a significant branch of comparative linguistics ${ }^{66}$, comparative linguistics qui satisfait à la fois à la dimension dynamique du processus de traduction bilatérale et qui est comprise, bien entendu, dans le sens que j'ai essayé de délimiter.

K.-RICHARD BAUSCH

64. Comme T. Krzeszowski le propose : " Fundamental Principles of Structural Constrastive Studies ", Glottodidactica, vol. II (1967), p. 33; cf. aussi la section "Theory of Translation » de l'Association internationale de linguistique appliquée (AILA).

65. Cf. aussi J. C. Catford, A Linguistic Theory of Translation, An Essay in Applied Linguistics, Londres, 1965, p. $19:$ "... the theory of translation is essentially a theory of applied linguistics»; cf. aussi M. A. K. Halliday, "Comparison and Translation", The Linguistic Sciences and Language Teaching, Londres, 1964, p. 114 : "the theory of translation is an important aspect of general linguistics $》$.

66. Cf. par exemple E. A. Nida, "Science of Translation », Language, 45 (1969), p. 483-498. 